\title{
Evaluation of Direct Susceptibility Testing for Monomicrobial Growth from Bactec Blood Culture Bottles- A Pilot Study
}

\author{
Authors \\ Roshni K J*, Harisree S, Baijayanti M \\ Department of Microbiology, St Johns Medical College, Bangalore, Karnataka, India \\ *Corresponding author \\ Dr. Roshni K Jose \\ Department of Microbiology, St John's Medical College, Bangalore, Karnataka, India \\ Email: rkjose4665@gmail.com
}

\begin{abstract}
Background: Conventional methods for isolation and antibiotic susceptibility reports of bacterial isolates from positive blood culture bottles takes at least 48 hours. Direct susceptibility testing (DST) helps reduce the time to AST results by 24 hours enabling early initiation of treatment.

Materials and Methods: Blood cultures flagged positive by the Bactec or BacT ALERT systems between July and October 2016 were analyzed by Grams stain. Direct susceptibility test was performed on the broths with monomicrobial growth using the guidelines prescribed by British Society for Antimicrobial Chemotherapy (BSAC). Simultaneously routine culture and susceptibility testing were also carried out for all the isolates.

Results: Both gram negative and gram positive organisms were subjected to DST. Out of 30 Gram positive isolates subjected to DST, $(n=6)$ were Enterococcus species and $(n=24)$ Staphylococcus species. Out of 30 Gram negative isolates subjected to DST,the species tested were Klebsiella spp $(n=6)$, Escherichia coli $(n=5)$, Acinetobacter spp ( $n=7)$, Pseudomonas $(n=1)$, Morganella $(n=1)$, Salmonella spp $(n=10)$ in number.

Conclusions: Direct susceptibility testing enables clinicians to start antimicrobial therapy 24hrs earlier than the routine culture and susceptibility testing, thus reducing the mortality and morbidity in patients with blood stream infections. A high level of concordance was seen between DST and reference method.

Keywords: antimicrobial resistance, direct susceptibility, blood culture, monomicrobial, sepsis.
\end{abstract}

\section{Introduction}

Sepsis is one of the major causes of mortality and morbidity in hospitalized patients. At least $2 \%$ of hospitalized patients suffer from blood stream infections. $^{(1)}$ Mortality is high especially in intensive care patients with septicemia. There is an increase in mortality by about $7.6 \%$ every hour of delay in initiation of antimicrobial therapy. ${ }^{(2)}$ Hence early initiation of appropriate antibiotic therapy helps reduce the mortality and morbidity in patients with septicemia. ${ }^{(3),(4)}$ Prompt and quick identification of bacteremia in patients especially in ICU settings lead to a more targeted antibiotic therapy. This helps reduce mortality and morbidity in such patients. A more targeted empirical therapy also helps reduce the development of antimicrobial resistance in patients with blood stream infections. ${ }^{(4)}$ In a routine microbiology laboratory the turnaround time for antimicrobial sensitivity testing (AST) results from positive blood culture bottles is about 
48 hours as it involves Gram stain and subculture onto blood agar (BA) and MacConkey agar (MA) of the culture broth and overnight incubation which yield isolated colonies which are then subjected to identification and AST by manual or automated methods. Direct susceptibility testing (DST) is a well-established method for diagnosis of septicemia. (5),(6) Various studies assessing the outcome of early initiation of appropriate antimicrobial therapy following rapid reporting of AST results have shown the decrease in the mortality and morbidity rates in septicaemic patients with decreased length of stay and treatment costs. ${ }^{(7),(8)}$ DST by disk diffusion method has added the advantage of finding clones of bacteria identical in colony morphology and biochemical characteristics but different in antibiotic susceptibility. ${ }^{(9)}$ DST will also help in enforcing antimicrobial stewardship practices in a tertiary care center thereby reducing the treatment costs and development of antimicrobial resistance due to inappropriate antimicrobial therapy.

\section{Materials and Methods}

This was a prospective study, from July 1st, 2016 to Oct 31st, 2016, for a period of 4 months. Blood cultures flagged positive by the BACTEC 9120 or BacT/Alert systems were analyzed by Grams stain. Those samples with polymicrobial growth as assessed by Grams stain were excluded from the study. Direct susceptibility test was performed on the broths with monomicrobial growth using the British Society for Antimicrobial Chemotherapy (BSAC) guidelines for both Gram negative and Gram positive bacteria. (10) A sterile 20 gauge needle was fitted onto the disposable syringe and used to extract the blood culture broth from the bottles with all sterile precautions.

- Based on the gram smear morphology- GPC in clusters-Staphylococcus species, GPC in pairs and short chains- Enterococcus species

- For Staphylococcus and Enterococcus using a 20 gauge needle, three drops of the broth were diluted in $5 \mathrm{~mL}$ of sterile distilled water and this was used to inoculate Mueller
Hinton Agar (MHA) plates. This dilution resulted in a semi confluent growth on MHA

- For Gram negative bacteria, a 20 gauge needle was used and one drop of the broth was diluted in $5 \mathrm{~mL}$ of sterile distilled water and this was used to inoculate MHA plates. This dilution resulted in a semi confluent growth on MHA.

- Samples were excluded from the study if the inoculum was not enough and growth was not semi confluent, or the culture was mixed showing polymicrobial growth.

- Simultaneously identification and Antibiotic Susceptibility Testing (AST) of all isolates were performed with by VITEK-2 or manual methods after overnight subculture of the blood culture broth which yielded pure growth and was compared.

The following definitions were adopted to analyze the results

$>$ Minor error-reference method sensitive(S)or resistant $(\mathrm{R})$ and test method is intermediate(I)or vice versa

$>$ No error-Test method and Reference method are same

Major error-Reference method is $\mathrm{S}$ and Test method is $\mathrm{R}$

$>$ Very major error-Reference method is R and Test method is $\mathrm{S}$

\section{Results}

A total of 200 blood cultures were analyzed of which 60 blood culture bottles yielded monomicrobial growth as per Gram stain analysis. Of the 60, 30 were gram positive cocci and 30 were Gram negative bacilli. Of the 30 Gram positive cocci 6 were identified as Enterococcus species and 24 as Staphylococcus species. Of the 30 Gram negative bacilli the various bacteria analyzed were Klebsiella spp (n-6), Escherichia coli (n-5), Acinetobacter spp (n-7), Pseudomonas spp (n-1), Morganella (n-1), Salmonella spp (n-10) in number. 
Table 1: Sensitivity results of Enterococcus species

\begin{tabular}{|l|c|c|c|c|}
\hline Drug & $\begin{array}{c}\text { No } \\
\text { error }\end{array}$ & $\begin{array}{c}\text { Minor } \\
\text { errors }\end{array}$ & $\begin{array}{c}\text { Major } \\
\text { errors }\end{array}$ & $\begin{array}{c}\text { Very } \\
\text { major } \\
\text { errors }\end{array}$ \\
\hline Penicillin (10U) & $83.3 \%$ & NIL & NIL & $16.6 \%$ \\
\hline $\begin{array}{l}\text { Erythromycin } \\
(15 \mu \mathrm{g})\end{array}$ & $83.3 \%$ & NIL & NIL & $16.6 \%$ \\
\hline $\begin{array}{l}\text { High-level } \\
\text { Gentamicin } \\
(120 \mu \mathrm{g})\end{array}$ & $100 \%$ & NIL & NIL & NIL \\
\hline
\end{tabular}

Table 2: Sensitivity results of Staphylococcus species

\begin{tabular}{|l|c|c|c|c|}
\hline Drug & $\begin{array}{c}\text { No } \\
\text { error }\end{array}$ & $\begin{array}{c}\text { Minor } \\
\text { errors }\end{array}$ & $\begin{array}{c}\text { Major } \\
\text { errors }\end{array}$ & $\begin{array}{c}\text { Very major } \\
\text { errors }\end{array}$ \\
\hline Penicillin (10U) & $87.5 \%$ & NIL & $8.3 \%$ & $4.1 \%$ \\
\hline $\begin{array}{l}\text { Erythromycin } \\
(15 \mu \mathrm{g})\end{array}$ & $87.5 \%$ & NIL & $8.3 \%$ & $4.1 \%$ \\
\hline $\begin{array}{l}\text { Gentamicin } \\
(10 \mu \mathrm{g})\end{array}$ & $91.6 \%$ & NIL & $8.3 \%$ & NIL \\
\hline Cefoxitin $(30 \mu \mathrm{g})$ & $83.3 \%$ & NIL & $4.1 \%$ & $12.5 \%$ \\
\hline $\begin{array}{l}\text { Tetracycline } \\
(30 \mu \mathrm{g})\end{array}$ & $83.3 \%$ & NIL & $4.1 \%$ & $12.5 \%$ \\
\hline
\end{tabular}

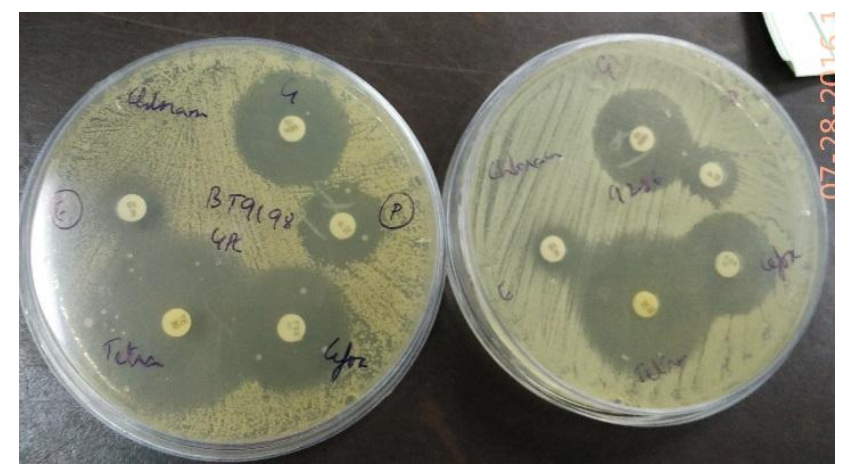

Fig 1 Direct Antibiotic Susceptibility plate showing Gram positive cocci from Blood Culture Broth Species distribution of various Gram negative organisms included in the study were Klebsiella spp $20 \%$ (n-6), Escherichia coli 16.6\% (n-5), Acinetobacter spp 23\% (n-7), Pseudomonas spp 3.3\% (n-1), Morganella 3.3\% (n-1), Salmonella spp 33.3\% (n-10)

Table 3: Sensitivity results of Gram negative bacteria

\begin{tabular}{|l|c|c|c|c|}
\hline Drug & $\begin{array}{c}\text { No } \\
\text { error }\end{array}$ & $\begin{array}{c}\text { Minor } \\
\text { errors }\end{array}$ & $\begin{array}{c}\text { Major } \\
\text { errors }\end{array}$ & $\begin{array}{c}\text { Very } \\
\text { major } \\
\text { errors }\end{array}$ \\
\hline Gentamicin $(10 \mu \mathrm{g})$ & $93.3 \%$ & $3.3 \%$ & $3.3 \%$ & NIL \\
\hline Meropenem $(10 \mu \mathrm{g})$ & $100 \%$ & NIL & NIL & NIL \\
\hline $\begin{array}{l}\text { Piperacillin-tazobactam } \\
(100 / 10 \mu \mathrm{g})\end{array}$ & $90 \%$ & $3.3 \%$ & NIL & $6.6 \%$ \\
\hline Ceftriaxone $(30 \mu \mathrm{g})$ & $90 \%$ & NIL & NIL & $10 \%$ \\
\hline Ciprofloxacin $(5 \mu \mathrm{g})$ & $80 \%$ & $10 \%$ & $3.3 \%$ & $6.6 \%$ \\
\hline
\end{tabular}

$>$ In Enterobacteriaceae $(\mathrm{n}=22)$, a very major error was found for Piperacillin Tazobactam (4.5\%), Ceftriaxone (13.63\%) and Ciprofloxacin $(9.09 \%)$. One major error was for Gentamicin (4.5\%) and Ciprofloxacin (4.5\%). One minor error for Gentamicin $(4.5 \%)$ and Ciprofloxacin $(9.09 \%)$

$>$ In non Enterobacteriaceae $(n=8)$, very major error was found for Piperacillin Tazobactam (4.5\%) and Ciprofloxacin (4.5\%), One major error and one minor error was for Ciprofloxacin (4.5\%).

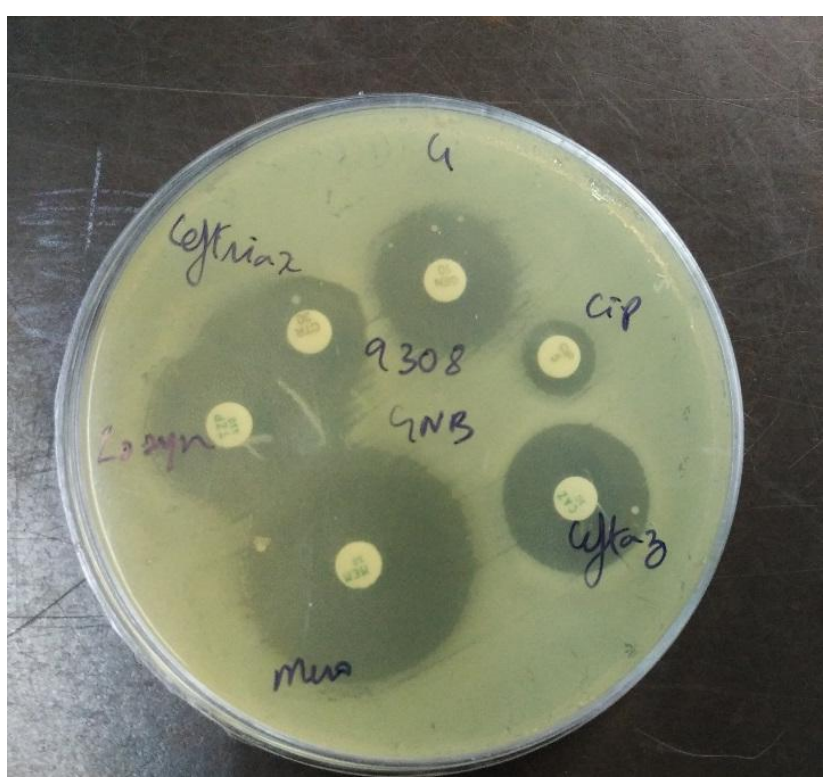

Fig 2 Direct Antibiotic Susceptibility plate showing Gram negative bacilli from Blood Culture Broth

\section{Discussion}

Standardization of the inoculum to be used for susceptibility testing was the major challenge during this study. We observed semi confluent bacterial growth on MHA plates once the inoculum was standardized. There is high concordance between DST and final AST (>80\%). Shortening of time to results of susceptibility testing of blood culture isolates can lead to significant reductions in patient morbidity, mortality, and costs. In a similar study by Goel et al, it has been shown that DST and AST are highly concordant and helps reduce mortality in septicemic patients. ${ }^{(11)}$

The errors in the sensitivity results are probably due to lack of standardization in the inoculum size and 
inability to obtain similar looking colonies to make inoculum for DST as is done for routine AST by disk diffusion method and VITEK 2 methods and further studies are required to evaluate the application of this in routine practice.

In this study, the antimicrobials like Vancomycin and Colistin have not been considered as there are no disc diffusion criteria for these drugs to compare. During the course of this study, it was observed that DST is comparable with AST/ Vitek 2 in case of Gram negative bacilli than Gram positive cocci which had more number of errors.

Standardization of inoculum for Drug susceptibility testing is the most critical step to achieve a semi confluent growth on the Muller Hinton Agar plate. In a study by Fay and Oldfather, the importance of standardizing the inoculum for direct susceptibility testing has been tackled. Hence, more extensive studies are required to standardize inoculum size for DST. The proper dilution of the inoculum in sterile saline and also the proper choice of antimicrobials will enable the application of Direct Susceptibility testing in routine laboratory testing.

\section{Conclusion}

Based on this study and its results, direct susceptibility testing enables clinicians to start empirical antimicrobial therapy $24 \mathrm{hrs}$ earlier than the routine culture and susceptibility testing, thus reducing the mortality and morbidity in patients with blood stream infections.

\section{References}

1. Angus DC, Linde-Zwirble WT, Lidicker J, Clermont G, Carcillo J, Pinsky MR. Epidemiology of severe sepsis in the United States: Analysis of incidence, outcome, and associated costs of care. Crit Care Med 2001;29:1303-10

2. Kumar A, Roberts D, Wood KE, Light B, Parrillo JE, Sharma S, et al. Duration of hypotension before initiation of effective antimicrobial therapy is the critical determinant of survival in human septic shock. Crit Care Med 2006;34:1589-96.
3. Bearman GM, Wenzel RP. Bacteremias: A leading cause of death. Arch Med Res 2005;36:646-59.

4. Fraser A, Paul M, Almanasreh N, Tacconelli E, Frank U, Cauda R, et al. TREAT Study Group. Benefit of appropriate empirical antibiotic treatment: Thirty-day mortality and duration of hospital stay. Am J Med 2006;119:970-6

5. Baron EJ, Weinstein MP, Dume WM, Yagupsky P, Welch DF, Wilson DM, et al. Blood Cultures IV. Washington: American Society of Microbiology; 2005.

6. Gherardi G, Angeletti S, Panitti M, Pompilio A, Di Bonaventura G, Crea F, et al. Comparative evaluation of the Vitek-2 Compact and Phoenix systems for rapid identification and antibiotic susceptibility testing directly from blood cultures of Gramnegative and Gram positive isolates. Diagn Microbiol Infect Dis 2012;72:20-31.

7. Barenfanger J, Drake C, Kacich G. Clinical and financial benefits of rapid bacterial identification and antimicrobial susceptibility testing. J Clin Microbiol 1999;37:1415

8. Doern GV, Vautour R, Gaudet M, Levy B. Clinical impact of rapid in vitro susceptibility testing and bacterial identification. $\mathrm{J}$ Clin Microbiol 1994;32:1757-62

9. Bhattacharya S. Early diagnosis of resistant pathogens: How can it improve antimicrobial treatment? Virulence 2013;4:172-84.

10. J M Andrews. BSAC standardized disc susceptibility testing method (version 5); Journal of Antimicrobial Chemotherapy: July 2006

11. G Goel, D Das, S Mukherjee et al., A method for early detection of antibiotic resistance in positive blood cultures: experience from an oncology centre in eastern India, IJMM 2015: S53-58

12. Fay D, Oldfather JE. Standardization of direct susceptibility test for blood cultures. J Clin Microbiol 1979; 9: 347-50. 